\title{
Characterization of immunoglobulin $\mathbf{G}$ antibodies to Plasmodium falciparum sporozoite surface antigen $M_{2}$ in malaria exposed individuals
}

\author{
Thanh V Nguyen ${ }^{1,2}$, John B Sacci Jr ${ }^{3}$, Patricia de la Vega ${ }^{3,4}$, Chandy C John ${ }^{5}$, \\ Anthony A James ${ }^{1,6}$ and Angray S Kang*7
}

\begin{abstract}
Address: ${ }^{1}$ Department of Molecular Biology and Biochemistry, University of California, Irvine, CA 92697, USA, ${ }^{2}$ NeoGenomics California, 6 Morgan, Suite 150, Irvine, CA 92618, USA, ${ }^{3}$ Department of Microbiology and Immunology, University of Maryland, School of Medicine, Room 324660 W Redwood Street, Baltimore, MD 21201, USA, ${ }^{4}$ Department of Cell Mediated Immunity, Division of Malaria Vaccine Development, US Military Malaria Vaccine Program, Walter Reed Army Institute of Research, USA, ${ }^{5}$ Global Pediatrics Program and Division of Pediatric Infectious Diseases, University of MN Medical School, 420 Delaware Street, SE, MMC \#296, 850-Mayo, Minneapolis, MN 55455, USA, ${ }^{\circ}$ Department of Microbiology and Molecular Genetics, University of California, Irvine, CA 92697-3900, USA and 7The School of Life Sciences, Department of Molecular and Applied Biosciences, University of Westminster, 115 New Cavendish Street, London, W1W 6UW, UK
\end{abstract}

Email: Thanh V Nguyen - quangson@yahoo.com; John B Sacci - jsacc001@umaryland.edu; Patricia de la Vega - patricia.delavega@us.army.mil; Chandy C John - ccj@umn.edu; Anthony A James - aajames@uci.edu; Angray S Kang* - kanga@wmin.ac.uk

* Corresponding author

Published: 23 October 2009

Malaria Journal 2009, 8:235 doi:10.1 I86/1475-2875-8-235
Received: 26 June 2009

Accepted: 23 October 2009

This article is available from: http://www.malariajournal.com/content/8/I/235

(c) 2009 Nguyen et al; licensee BioMed Central Ltd.

This is an Open Access article distributed under the terms of the Creative Commons Attribution License (http://creativecommons.org/licenses/by/2.0), which permits unrestricted use, distribution, and reproduction in any medium, provided the original work is properly cited.

\begin{abstract}
Background: MB2 protein is a sporozoite surface antigen on the human malaria parasite Plasmodium falciparum. MB2 was identified by screening a $P$. falciparum sporozoite cDNA expression library using immune sera from a protected donor immunized via the bites of $P$. falciparum-infected irradiated mosquitoes. It is not known whether natural exposure to $P$. falciparum also induces the anti-MB2 response and if this response differs from that in protected individuals immunized via the bites of $P$. falciparum infected irradiated mosquitoes. The anti-MB2 antibody response may be part of a robust protective response against the sporozoite.

Methods: Fragments of polypeptide regions of MB2 were constructed as recombinant fusions sandwiched between glutathione S-transferase and a hexa histidine tag for bacterial expression. The hexa histidine tag affinity purified proteins were used to immunize rabbits and the polyclonal sera evaluated in an in vitro inhibition of sporozoite invasion assay. The proteins were also used in immunoblots with sera from a limited number of donors immunized via the bites of $P$. falciparum infected irradiated mosquitoes and plasma and serum obtained from naturally exposed individuals in Kenya.
\end{abstract}

Results: Rabbit polyclonal antibodies targeting the non-repeat region of the basic domain of MB2 inhibited sporozoites entry into HepG2-A16 cells in vitro. Analysis of serum from five human volunteers that were immunized via the bites of $P$. falciparum infected irradiated mosquitoes that developed immunity and were completely protected against subsequent challenge with nonirradiated parasite also had detectable levels of antibody against MB2 basic domain. In contrast, in three volunteers not protected, anti-MB2 antibodies were below the level of detection. Sera from protected volunteers preferentially recognized a non-repeat region of the basic domain of MB2, whereas plasma from naturally-infected individuals also had antibodies that recognize regions of 
MB2 that contain a repeat motif in immunoblots. Sequence analysis of eleven field isolates and four laboratory strains showed that these antigenic regions of the basic domain of the MB2 gene are highly conserved in parasites obtained from different parts of the world. Moreover, anti-MB2 antibodies also were detected in the plasma of $83 \%$ of the individuals living in a malaria endemic area of Kenya $(n=4 I)$.

Conclusion: A preliminary analysis of the human humoral response against MB2 indicates that it may be an additional highly conserved target for immune intervention at the pre-erythrocytic stage of $P$. falciparum life cycle.

\section{Background}

Parasites of the Plasmodium species that are transmitted to people through the bites of infected mosquitoes cause malaria, a life-threatening disease. Malaria poses a serious public health problem in many parts of the world and approximately half of the world's population is at risk, in particular those living in lower-income countries [1]. The four types of human malaria are caused by Plasmodium falciparum, Plasmodium vivax, Plasmodium malariae and Plasmodium ovale. Of these, $P$. falciparum and $P$. vivax are the most common and P. falciparum is the most deadly [1]. Emergence of drug and insecticide resistance has exacerbated the situation, undermining the effectiveness of existing malaria control methods that depend on chemotherapy and vector control, respectively. Clearly, additional effective means to fight the disease, such as a safe and effective vaccine(s) are needed urgently. Currently, several approaches to developing malaria vaccine are in various stages of pre-clinical and clinical development involving single and multi-stage targets these are discussed in depth elsewhere [2-6].

Successful vaccination of humans on a limited scale against $P$. falciparum malaria was achieved first using irradiated sporozoites as an immunogen [7]. This approach follows the classical route of vaccine development via attenuation; in this case radiation induced attenuation resulting in non-replicating metabolically-active $P$ falciparum sporozoites and results in targeting the pre-erythrocytic stage. This type of vaccine has to be $100 \%$ effective to induce sterile protective immunity and prevent the development of blood-stage infection in naïve individuals. Other vaccine candidates targeting the pre-erythrocytic stage that are less than $100 \%$ effective, may not prevent, but delay the onset of disease in naïve individuals and reduce subsequent episodes of clinical malaria [8], and as such may still play an important role in the fight against malaria. Although non-replicating metabolically-active sporozoites as immunogen(s) appears to be effective and the limited data are encouraging, the development of this approach leading to a licensed product for the prevention of malaria infection presents challenges and opportunities [9]. As efforts continue to develop this potential preerythrocytic stage attenuated vaccine, the volunteers that have already participated in the early phases of validation warrant further evaluation to examine the nature of this induced sterile protective response with a view to identifying key responsive elements to provide insights into the molecular basis of this immunity.

The pre-erythrocytic immune response is primarily directed against the circumsporozoite (CS) protein, a surface protein of Plasmodium sporozoites [10-12]. The CS protein is a leading vaccine candidate because irradiated sporozoite-induced protection in volunteers correlates with high circulating levels of anti-CS antibodies [13], and these antibodies are directed against the immunodominant B cell epitopes in the central tetramer repeat [Asparagine Proline Asparagine Alanine $]_{n}(\mathrm{NPNA})_{n}$. Moreover a human monoclonal antibody directed against CS protein $(\mathrm{NPNA})_{\mathrm{n}}$ tetramer repeat isolated from a protected individual immunized via bites of infected, irradiated mosquitoes and subsequently shown to be protected against non-irradiated parasite challenge exhibited dose-dependent inhibition of $P$. falciparum sporozoites invasion of HepG2-A16 cells in vitro $[14,15]$. Recent attempts to induce protection in humans using $P$. falciparum CS-based vaccines containing the NPNA repeat motif, with improvement in their immunogenicity, and formulation, such as RTS, S/AS02 have yielded promising results [16]. To induce the protective immunity that is as consistent and long-lasting as that observed in the irradiated-sporozoite exposed individuals may require a vaccine that not only targets additional sporozoites surface antigens, but also provides a mechanism for extended priming of the immune system. The later present significant challenges in vaccine delivery and short of radiation or geneticallyattenuated non-replicating metabolically-active sporozoite, may be difficult to mimic. However, in addition to the immunodominant CS protein it may be important to identify antigens that may act independently, additively or synergistically and contribute towards more robust protection. To-date several candidate molecules, including thrombospondin-related anonymous protein (TRAP)[17], also know as sporozoite surface protein 2 (SSP2)[18], sporozoite-threonine-asparagine-rich protein (STARP)[19] and the more recently identified MB2 [20], have been shown to be present on the sporozoite surface. 
The P. falciparum gene MB2, is a single copy gene on chromosome five, and is expressed as a single mRNA transcript in erythrocytic stage of parasites, the predicted translational product is 1610 amino acids in length with a predicted mass of $187 \mathrm{kDa}$. The translated primary sequence may be further subdivided into three domains, an amino terminal basic domain, a central acidic domain and a carboxyl terminal domain with high sequence similarity to GTP-binding domains. The MB2 gene products are present in the sporozoite, asexual blood stages and gametocytes and have a distinct pattern of stage dependent subcellular localisation and proteolytic processing at the various stages of the parasite life cycle[20]. To-date gene homologues to the P. falciparum MB2, (PfMB2) have been identified in Plasmodium knowlesi(PkMB2), Plasmodium gallinaceum (PgMB2), Plasmodium berghei (PbMB2), Plasmodium yoelii (PyMB2), Plasmodium chabaudi (PcMB2) [21] and $P$. vivax(PvMB2) (GenBank Accession $\underline{\mathrm{XM} 001613742)}$. Of four conserved regions of the putative MB2 translation products, two have similarity to known proteins, S1 domain involved in initiation of translation and mRNA turnover and a GTP-binding domain similar to the family G-domains involved in protein synthesis [21]. In the non-conserved region of PfMB2, $P y M B 2$ and $P g M B 2$ tandemly repeated amino acid motifs are present. Repeat motifs are present in other malarial surface proteins including CS, TRAP and merozoite surface proteins (MSP) [22]. These repetive regions are T-cell independent repeat regions of parasite antigen and it has been postulated that the immune response against these regions diverts the response away from more important epitopes [23].

The MB2 antigen possesses intriguing immunogenic and molecular properties that indicate that it may be an important immune target in the non-replicating metabolically active $P$. falciparum sporozoite attenuated vaccine and may complement and enhance the efficacy of preerythrocytic stage vaccines currently in development.

\section{Methods}

\section{Recombinant protein expression and purification}

Fragments of the MB2 open reading frame were expressed in bacteria as Glutathione-S-Transferase (GST)-MB26xHistidine (His) fusion proteins in the dual-affinity pAK1-6H expression vector [24]. The oligonucleotide primers used to construct the GST-MB2-6xHis recombinant proteins are listed in Table 1 . The cloning of MB2 fragments into the expression vector was described in earlier studies [20]. Purification of recombinant proteins was carried out using the ProBond resin (Invitrogen) with the addition of imidazole at $85 \mathrm{mM}$ final concentration in the

Table I: Names, positions, and sequences of oligonucleotide primers used to clone and express GST-fusion proteins representing various regions of the MB2 open reading frame.

\begin{tabular}{|c|c|}
\hline GST-fusion & Amino acida $^{\mathrm{a}}$ \\
\hline \multicolumn{2}{|l|}{ MB2-A } \\
\hline \multicolumn{2}{|l|}{5 ' primer-Nco I b(98)GATGCCATGGGTGTTAATAGATGTTTTAC } \\
\hline $\begin{array}{l}\text { 3' primer-Sma I (305)GATCCCGGGGAGCATATTCTATTATATTCA } \\
\text { MB2-B }\end{array}$ & $32-101$ \\
\hline \multicolumn{2}{|l|}{ 5' primer-Nco I (286)GATGCCATGGAATATAATAGAATATGCA } \\
\hline 3' primer-Sma I (620)GATCCCGGGTTTTTATTATTAGAAGAATCA & $95-206$ \\
\hline \multicolumn{2}{|l|}{ MB2-Cc } \\
\hline \multicolumn{2}{|l|}{ 5' primer-Nco I (602)GATGCCATGGATTCTTCTAATAATAAAAT } \\
\hline 3' primer-Sma I (953)ATGCATCCCCGGGTCATTTTTTATTTGAAGAATTCTC & $200-316$ \\
\hline \multicolumn{2}{|l|}{ MB2-Fd } \\
\hline \multicolumn{2}{|l|}{ 5' primer-Nco I (98)GATGCCATGGGTGTTAATAGATGTTTTATC } \\
\hline 3' primer-Sma I (953)ATGCATCCCCGGGTCTTTTTTATTTGAAGAATTCTC & $32-316$ \\
\hline \multicolumn{2}{|l|}{ MB2-D } \\
\hline \multicolumn{2}{|l|}{ 5' primer-Nco I (1066)GATGCCATGGCATCTACATTAGATGAAACA } \\
\hline 3' primer-Sma I (1640)GATCCCGGGGATGTACTATAATCATTATTTGG & $355-546$ \\
\hline \multicolumn{2}{|l|}{ MB2-E } \\
\hline \multicolumn{2}{|l|}{ 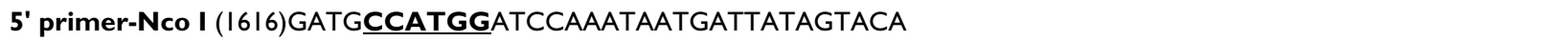 } \\
\hline $\begin{array}{l}\text { 3' primer-Sma I (232I)GATCCCGGGCTTGAATTATATTCTTTATTTTCGTG } \\
\text { MB2-FAc }\end{array}$ & $538-773$ \\
\hline \multicolumn{2}{|l|}{ 5' primer-Nco I (2294)GTATGCCATGGTCCACGAAAATAAAGAATATAATTCAAG } \\
\hline 3' primer-Sma I (2837)GATCCCGGGTCATCGAGCGATTCATTTTGGTC & $764-945$ \\
\hline \multicolumn{2}{|l|}{ MB2-IF2 } \\
\hline \multicolumn{2}{|l|}{5 ' primer-Nco I (4009)GATGCCATGGATGGTAATAGAACAAATAATGAC } \\
\hline 3' primer-Sma I (4823)GATCCCGGGTACGCTTCGATTATATCGTTTGGCTC & $1337-1606$ \\
\hline
\end{tabular}

a Numbers indicate amino acid positions relative to the initiation methionine (I), b Numbers in parenthesis refer to nucleotide positions relative to the ATG initiation codon; bold underlined letters indicate the restriction endonuclease sites, Nco I and Sma I,c These peptides includes amino acid repeats, ${ }^{d}$ This peptide is a combination of $M B 2-A,-B$, and $-C$. 
washing buffer. Eluted fractions were analysed by SDSPAGE and immunoblotting using anti-GST antibodies.

\section{Human plasma and serum}

Samples of plasma were obtained from individuals living in an area of Kenya with highly seasonal malaria transmission. Samples were obtained during high transmission season from individuals two years of age or older. Plasma donors were assessed for symptoms of malaria and the presence of $P$. falciparum parasitaemia on microscopic examination of peripheral blood smears. Of the forty-one plasma donors, nine were smear negative, seven were smear positive but asymptomatic, and twenty-five were smear positive and symptomatic. Symptomatic individuals had one or more of the following symptoms: fever, chills, headache, severe malaise or vomiting. Samples with available serum were tested for antibodies to CSP and TRAP by ELISA, as described previously [25]. Levels were given in arbitrary units (AU). The AU was determined by dividing the optical density (OD) of the study sample by the mean plus three standard deviations of the ODs of nine North American control sera. The cut-off for a positive response was an AU of 1 . Informed consent was obtained from all individuals and their guardians, as described previously [26]. Ethical approval was obtained from the Ethical Review Committee at the Kenya Medical Research Institute and the Human Investigations Institutional Review Board at Case Western Reserve University and the University Hospital of Cleveland.

Samples of serum from eight volunteers experimentally immunized by the bites of irradiated, infected mosquitoes were obtained from W.O. Rogers at the US Naval Medical Research Center (Rockville, Maryland); and from U. Krzych at the Walter Reed Army Institute of Research (Washington DC). Upon challenge with bites of P. falciparum infected, non-irradiated mosquitoes out of the eight volunteers five were completely protected (W. Rogers and U. Krzych, personal communication).

\section{Immunoblot analyses}

Purified GST-MB2 recombinant proteins ( $~ 50-100 \mathrm{ng}$ ) were resolved by SDS-PAGE in a $12 \%$ polyacrylamide gel and transferred onto a nitrocellulose membrane. The membrane was incubated with the human plasma or serum diluted 1:100 in Tris-buffered saline (TBS) + 0.05\% Tween-20 (TBST) for two hours at room temperature. Following washing with TBST, the filters were incubated in HRP-conjugated anti-human IgG (CalBiochem, San Diego, CA) diluted 1:80,000 in TBST and the enhanced chemiluminescence (ECL) system (Amersham Biosciences Corp, Piscataway, NY) used for detection. Rabbit anti-GST antibodies were used as a positive control and normal human serum was used as a negative control.

\section{Amplification and sequence of MB2 genes from diverse parasite populations}

Plasmodium falciparum genomic DNA extracted from laboratory-maintained strains and field isolates from infected red blood cells was kindly provided by Dr. A. Lal, (Centers for Disease Control and Prevention, Atlanta). The nucleotides encoding the antigenic region of the $\mathrm{B}$ domain of MB2 (aa 1-317 amino acids) were amplified from genomic DNA by the polymerase chain reaction (PCR) using the following primers: 5'-ATGTTTCTAATATGGCGTTTG-3' and 5'-TCATTTTTATTTGAAGAATT-3'. Conditions for amplification included an initial DNA denaturation of $94^{\circ} \mathrm{C}$ for $2 \mathrm{~min}$, followed by 30 cycles at $94^{\circ} \mathrm{C}$ for $20 \mathrm{~s}, 55^{\circ} \mathrm{C}$ for $20 \mathrm{~s}$, and $60^{\circ} \mathrm{C}$ for $1 \mathrm{~min}$. Genomic DNA (200 ng) of each lab strain was used as template in the reaction. The concentration of genomic DNA of field isolates used as template in the amplification reaction is not known due to the limited quantity of the sample. The amplification products were cloned into a TA cloning vector using the TOPO-PCR cloning kit (Invitrogen). Plasmid DNA was prepared from bacterial cultures and the inserts sequenced in both directions. Cloning and sequencing were repeated on amplification products obtained independently to ensure reproducibility of the sequence data. Alignment of the sequences was performed by the Clustal method using the Megalign program from the Lasergene computer software.

\section{Rabbit immunization and total IgG purification}

Rabbit immunization procedures used to obtain polyclonal antibodies against $\mathrm{MB} 2$ recombinant proteins are described elsewhere [20]. For IgG purification, the ImmunoPure ${ }^{\circledast}$ IgG Protein A purification kit was used following the protocol provided by the manufacturer (Pierce, Rockford, IL).

\section{In vitro inhibition of sporozoite invasion (ISI) assay}

To determine whether antibody targeting a particular domains or sub domains of MB2 had an effect on the ability of sporozoites to enter hepatocytes, total purified IgG from sera obtained from rabbits immunized against three different regions of $\mathrm{MB} 2$ (MB2-B, -C, and -FA) and a positive control NFS1 monoclonal antibody [27] were used in ISI assays of cultured liver cells [28,29]. A human hepatoma cell line, HepG2-A16, [28] was used for the assay. Briefly 50,000 HepG2-A16 cells were seeded on eight-chamber plastic Lab-Tek slides (Miles Research) in supplemented minimal essential medium (MEM) as described [28]. The purified antibodies were added to a final concentration of $100 \mu \mathrm{g} / \mathrm{ml}$, and HepG2-A16 cells were infected with 25,000 P. falciparum NF54 sporozoites. Slides were incubated at $37^{\circ} \mathrm{C}$ in $5 \% \mathrm{CO}_{2}$ for three hours, washed twice with phosphate-buffered saline (PBS), fixed with cold methanol, and rinsed twice again with PBS. Sporozoites that had invaded hepatoma cells were visualized 
by phase-contrast light microscopy using immunohistochemical staining with a mouse monoclonal antibody (NFS1) [27] against $P$. falciparum CS protein. Slides were incubated with NFS1 $(10 \mu \mathrm{g} / \mathrm{ml})$ for $30 \mathrm{~min}$ at room temperature, followed by goat anti-mouse IgG peroxidase conjugate for $30 \mathrm{~min}$ at room temperature. The ISI assays were done in triplicate with pre-immune IgG used as the negative control and the anti-CS monoclonal antibody NFS1 used as the positive control.

\section{Results}

\section{Recombinant protein expression and purification}

GST-MB2 recombinant proteins representing various regions of the coding sequence of $\mathrm{MB} 2$ were expressed in bacteria (Figure 1A). Bacteria transformed with plasmids carrying different regions of MB2 required different induction times and culture media for optimal yield (Table 2). The level of recovered protein varied greatly for each recombinant construct. The inclusion of $80-85 \mathrm{mM}$ imidazole in the wash buffer and an overnight wash during isolation were essential in improving the purity of the recombinant proteins. All of the expressed proteins were soluble and thus purified readily from the cell-free bacte- rial lysates by one-step nickel column chromatography and were readily detected in an immunoblot using rabbit anti-GST antibodies (Figure 1B).

\section{Immunoblot plasma and serum analyses}

All smear negative donors were exposed previously to $P$. falciparum as evidenced by the presence of anti-CS and anti-TRAP antibodies in their plasma (Table 3). The results of the immunoblot analyses obtained with the serum from a protected volunteer (\#5, Table 3) and endemic plasma (KU162, Table 3), showed that both contain antibodies against the basic domain and some residual antibody reactivity was observed against regions of the acidic domain, whilst no antibody reactivity was detected against the GTP binding domain fragment as shown in Figure 1. Moreover, the regions of MB2 reacting with the immune sera are limited to the central region of the basic domain in particular MB2-B and MB2-C fragments. It would appear that serum from this protected (\#5) individual reacts preferentially with MB2-B (non-repeat containing) and to a lesser degree with the MB2-C (repeat containing) region. In contrast the plasma from the endemic (KU162) region appears to have the inverse reac-

A

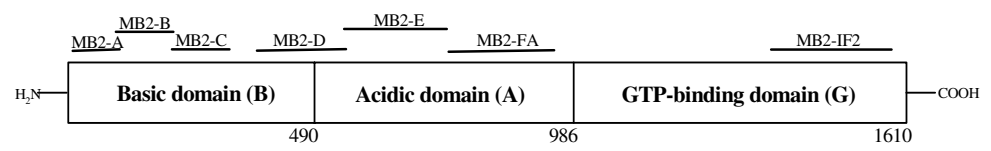

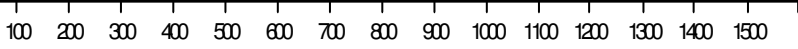

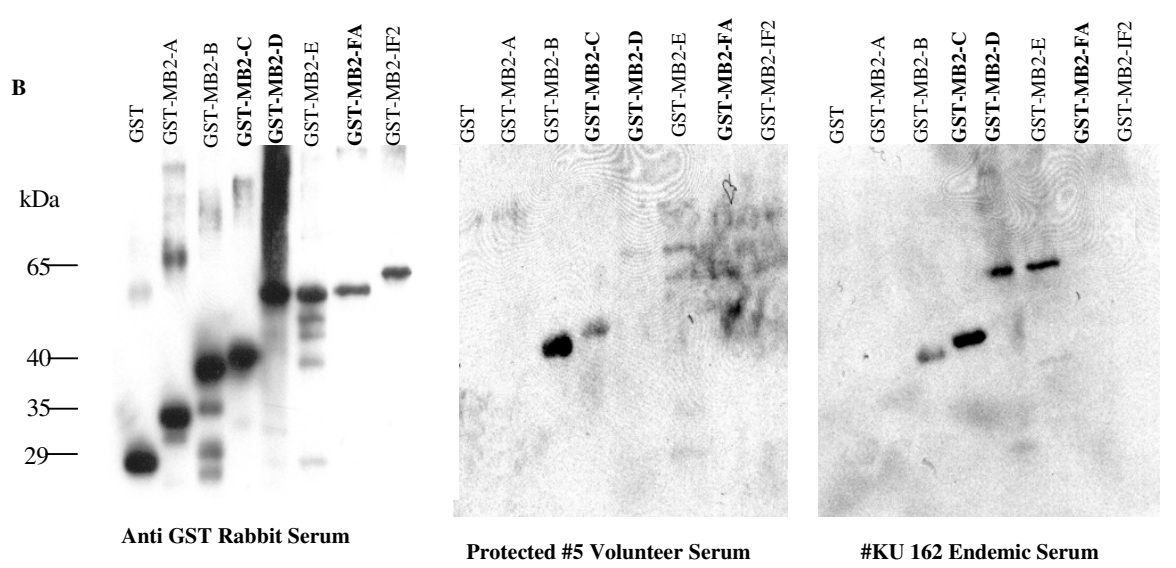

Figure I

Immunoblot analyses to assess the presence of antibody against MB2 recombinant peptides. A, is a schematic representation of the MB2 protein sequence. The three domains, Basic (B), Acidic (A), and GTP-binding (G), are indicated as blocks with the amino acid junctions numbered below. The seven short horizontal lines represent the approximate extents of the polypeptides that were expressed as GST-fusion recombinant proteins. B, immunoblots of GST-MB2 recombinant proteins reacted with anti-GST rabbit serum (Anti-GST); or serum of a protected volunteer (\#5 volunteer); or serum of a person living in a malaria-endemic area (Endemic serum \#KU I62). Immunoblots were prepared in triplicate, and each lane contains $50-100 \mathrm{ng}$ of purified GST-MB2 recombinant proteins. Recombinant proteins, MB2-C, MB2-D, and MB2-FA, listed in bold letters contain amino acid repeats. Approximate molecular weights of the fusion proteins are indicated in kilodaltons (kDa). 
Table 2: Optimal conditions to express GST-MB2 recombinant polypeptides in E. coli.

\begin{tabular}{|c|c|c|c|c|c|}
\hline Name & Amino Acid Positiona (Size) & Net Chargeb & Mediac $^{c}$ & Induction Time & Expression Leveld \\
\hline MB2-A & $32-101,(70 \mathrm{aa})$ & +8.94 & SB & $\mathrm{I} \mathrm{hr} 10 \mathrm{~min}$ & $\mathrm{l}-2 \mathrm{mg} / \mathrm{L}$ \\
\hline MB2-B & $95-206,(112 \mathrm{aa})$ & +10.18 & LB & $8 \mathrm{hrs}$ & $3 \mathrm{mg} / \mathrm{L}$ \\
\hline MB2-C & $200-316,(117 \mathrm{aa})$ & +2.58 & LB & $4 \mathrm{hrs}$ & $4 \mathrm{mg} / \mathrm{L}$ \\
\hline MB2-Fe(A-B-C) & $32-316,(285 \mathrm{aa})$ & +21.91 & SB & $4 \mathrm{hrs}$ & $2 \mathrm{mg} / \mathrm{L}$ \\
\hline MB2-D & $355-546,(192$ aa) & -0.72 & SB & I-6 hrs & $0.1-0.2 \mathrm{mg} / \mathrm{L}$ \\
\hline MB2-E & $538-773,(236 \mathrm{aa})$ & +8.23 & SB & I-2 hrs & $0.1-0.2 \mathrm{mg} / \mathrm{L}$ \\
\hline MB2-FA & $764-945,(182$ aa $)$ & $-|7.5|$ & SB & 3-6 hrs & $2 \mathrm{mg} / \mathrm{L}$ \\
\hline MB2-IF2 & $1337-1606,(270 \mathrm{aa})$ & +5.26 & LB or $S B$ & $\mathrm{l} \mathrm{hr}$ & $0.5-1.0 \mathrm{mg} / \mathrm{L}$ \\
\hline
\end{tabular}

a Position is based on the translation initiation methionine (I).

b Calculated at neutral $\mathrm{pH}$

c LB, Luria Broth; SB, Super Broth. Media were made according to instructions in Sambrook et al.,[39].

$d$ Expressed as amount of purified protein per volume of IPTG-induced culture

e Combined length of MB2-A+MB-2B+MB2-C

tivity (i.e., MB2-C staining more strongly than MB2-B). Moreover, the endemic plasma also contained antibodies against the acidic domain fragments MB2-D and MB2-E. The protected sera (\#5) had no detectable antibody against MB2-D, but some slight reactivity against MB2-E.

The initial immunoblot analysis showed that the protected volunteer (\#5) serum recognized principally the two regions in the basic domain, and these regions were recognized differently by the endemic (KU 162) plasma. To determine if this differential antibody recognition correlated with the different immunity observed between protected volunteers and the status of intermittentlyexposed individuals living in area of seasonal transmission, the immunoblot analysis was expanded to include plasma obtained from a total of forty one individuals living in an area of seasonal malaria transmission who were blood-smear negative for $P$. falciparum $(\mathrm{n}=9)$, blood smear positive but asymptomatic $(n=7)$ or blood smear positive and symptomatic $(\mathrm{n}=25)$. Serum samples from eight irradiated sporozoite exposed volunteers, of which five had acquired sterile immunity, were also included.

Results of the expanded immunoblot analyses of the basic domain fragments $\mathrm{MB} 2-\mathrm{A},-\mathrm{B}$ and $-\mathrm{C}$ are summarized in Table 3. A total of $83 \%$ (34/41) of naturally exposed individuals had antibodies against the basic domain of MB2 and prevalence against MB2-C and MB2-B was 78 and $61 \%$, respectively. The prevalence of antibody against $\mathrm{MB} 2-\mathrm{B}$ and - $\mathrm{C}$ in plasma from smear negative donors relative to smear positive donors was determined (MB2-B $89 \%, \mathrm{MB} 2-\mathrm{C}$ 100\%) and (MB2-B 53\%, MB2-C 72\%) respectively. The overall prevalence of antibody response against the repeat $M B 2-C$ region was greater than against the non-repeat, $\mathrm{MB} 2-\mathrm{B}$ region. Comparison between smear positive $(\mathrm{n}=32)$ (parasitaemic) and smear negative $(n=9)$ (non-parasitaemic) individuals for the presence of antibody against MB2-B approaches significance ( $\mathrm{p}=$ 0.066 , Fisher's exact test). Minimal or no antibody reac- tion was detected against the amino-terminal MB2-A region. In comparison, the immunoblot result obtained with sera of irradiated-sporozoite immunized volunteers showed an association between the anti-MB2 antibody response and the immune status of the volunteers (Table 3 ). The five protected volunteers produced anti-MB2 antibodies that were directed preferentially against the nonrepeat $\mathrm{MB} 2-\mathrm{B}$ region. In contrast, all three unprotected volunteers did not produce a detectable antibody response against $\mathrm{MB} 2$.

All nine smear negative individuals had antibodies to CSP and/or TRAP, demonstrating evidence of prior exposure to $P$. falciparum (Table 3 ). As compared to smear negative individuals, smear positive individuals who had plasma available for testing for antibodies to CSP and TRAP by ELISA had lower levels of antibodies to TRAP (smear positive, median AU 1.13, range 0.1 - 2.95, as compared to smear negative, median $\mathrm{AU} 2.08$, range, $1.17-4.8, \mathrm{P}=$ 0.02 ) but not CSP (smear positive, median AU 1.06, range 0.3 - 3.01, as compared to smear negative, median AU1.75, range, $0.55-4.28, \mathrm{P}=0.11)$. Smear positive individuals were also less frequently positive for antibodies to CSP (14 of 26 individuals tested) or TRAP (15 of 26 individuals tested) (Table 3 ).

\section{Polymorphism assessment of the antigenic region of the MB2 basic domain}

The nucleotide sequence encoding the first 317 amino acids of MB2 corresponding to the B-cell epitopes that were recognized differentially in the immunoblot analysis were determined. The nucleotide sequences have been deposited in GenBank with accession numbers AF454665, AF454666, AF454667, AF378132, AF378136 and XM 001351687. The translated amino acid sequence alignment of the antigenic region of MB2 from four laboratory strains and 11 field isolates collected from India ( $\mathrm{n}$ $=3)$, Venezuela $(n=4)$, Thailand $(n=3)$ and Papua New Guinea $(n=1)$ show that the only variation observed is in 
Table 3: Individual antibody responses to MB2 recombinant peptides of the B domain as determined by immunoblot analysis.

\begin{tabular}{|c|c|c|c|c|c|c|}
\hline & MB2-A ${ }^{a}$ & MB2-Ba & MB2-Ca & & & \\
\hline \multicolumn{7}{|l|}{ Volunteer serum } \\
\hline Volunteer \#I (protected) & $-b$ & $++b$ & $-/+b$ & & & \\
\hline Volunteer \#3 (protected) & - & ++ & $-/+$ & & & \\
\hline Volunteer \#5 (protected) & - & ++ & $-/+$ & & & \\
\hline Volunteer \#7 (protected) & - & ++ & $-/+$ & & & \\
\hline WRAIR \#I (protected) & - & ++ & $-/+$ & & & \\
\hline WRAIR \#4 (not protected) & - & - & - & & & \\
\hline WRAIR \#5 (not protected) & - & - & - & & & \\
\hline WRAIR \#6 (not protected) & - & - & - & & & \\
\hline $\begin{array}{l}\text { Kenyan serum } \\
\text { Smear negative }\end{array}$ & MB2-A & MB2-Ba & MB2-Ca & $\begin{array}{l}\text { Age } \\
\text { (yrs) }\end{array}$ & $\underset{A U^{d}}{\text { CSP }}$ & $\begin{array}{l}\text { TRAP } \\
\text { AUd }^{d}\end{array}$ \\
\hline c KU 036 & - & ++ & + & 29 & 1.77 & 2.35 \\
\hline KU 07I & - & ++ & +++ & 18 & 1.24 & 2.08 \\
\hline KU 08I & - & + & ++ & 34 & 1.01 & 1.23 \\
\hline KU 069 & - & + & ++ & 18 & 0.55 & 1.31 \\
\hline KU 079 & - & $-/+$ & ++ & 80 & 1.0 & 2.63 \\
\hline KU 083 & - & $-/+$ & +++ & 45 & 4.28 & 4.80 \\
\hline KU 163 & - & ++ & $-/+$ & 18 & 1.75 & 1.46 \\
\hline KU 202 & - & - & + & 30 & 2.22 & 2.22 \\
\hline KU 205 & - & $-/+$ & $-/+$ & 44 & 1.81 & 1.17 \\
\hline \multicolumn{7}{|c|}{ Smear positive, asymptomatic } \\
\hline $\mathrm{KU} / 18$ & - & - & - & 23 & 1.94 & 1.85 \\
\hline KU 044 & - & + & - & 6 & 0.75 & 0.79 \\
\hline KU 048 & - & - & + & 6 & 0.61 & 0.62 \\
\hline KU 076 & - & - & $-1+$ & 6 & 1.29 & 0.72 \\
\hline KU 001 & - & ++ & ++ & 2 & 1.11 & 1.43 \\
\hline KU 049 & - & - & - & 6 & 1.64 & 0.66 \\
\hline KU 025 & - & - & - & 6 & 0.42 & 0.26 \\
\hline \multicolumn{7}{|c|}{ Smear positive, symptomatic } \\
\hline KU 064 & - & - & - & 27 & 1.38 & 2.40 \\
\hline KU 070 & - & + & - & 18 & 1.41 & 1.59 \\
\hline KU I57 & - & + & +++ & 20 & 1.64 & 1.37 \\
\hline KU 158 & - & + & ++ & 26 & ND & ND \\
\hline KU 165 & - & - & - & 26 & 0.34 & 0.10 \\
\hline KU I72 & - & $-/+$ & ++ & 22 & ND & ND \\
\hline KU 199 & - & + & $-/+$ & 19 & ND & ND \\
\hline KU 203 & - & - & $-/+$ & 30 & 1.34 & 1.0 \\
\hline KU 207 & - & - & + & 19 & ND & ND \\
\hline KU 234 & - & + & $-/+$ & 42 & ND & ND \\
\hline KU 162 & - & + & +++ & 6 & ND & ND \\
\hline KU 062 & - & - & - & 6 & 0.87 & 1.85 \\
\hline KU I6I & - & - & $-/+$ & 7 & 0.67 & 0.88 \\
\hline KU 04I & - & - & $-1+$ & 6 & 1.62 & 0.71 \\
\hline KU 037 & - & ++ & $-/+$ & 60 & 1.36 & 1.52 \\
\hline KU 072 & - & $-/+$ & $-1+$ & 24 & 0.59 & 1.0 \\
\hline KU 075 & - & + & ++ & 32 & 0.72 & 1.35 \\
\hline KU 080 & - & $-/+$ & + & 34 & 0.87 & 1.50 \\
\hline KU 088 & - & - & - & 70 & 1.01 & 0.57 \\
\hline KU I45 & - & + & + & 40 & 3.01 & 2.56 \\
\hline KU I55 & - & - & ++ & 37 & 0.53 & 0.38 \\
\hline KU 174 & - & + & + & 22 & 2.27 & 1.90 \\
\hline KU 183 & - & $-1+$ & ++ & 23 & 0.30 & 0.36 \\
\hline KU 067 & - & - & + & 6 & 1.90 & 2.95 \\
\hline KU 084 & - & ++ & + & 2 & 0.40 & 1.277 \\
\hline
\end{tabular}

a Peptides are described in Figure I. ${ }^{~}-$ no detectable band, $-/+$ weak band but visible, + weak band, ++ moderate to strong band and +++ intense band on western blot. ${ }^{\circ} \mathrm{KU}=$ serum obtained from individuals living in Kenya ${ }^{\mathrm{d}} \mathrm{AU}=$ arbitrary units, see Methods for definition. CSP = circumsporozoite protein; TRAP $=$ thrombospondin related adhesive protein.

ND = not determined 


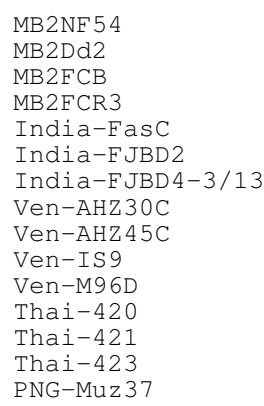

\section{Figure 2}

Amino acid sequence alignment showing the size polymorphism in the repeat region of the MB2 gene from different laboratory strains and field isolates. Amino acid positions $2 \mathrm{II}$ to 264 make up the repeat domain. Identical amino acids outside the repeat domain are not shown. Field isolates were surveyed from India, Venezuela (Ven), Thailand (Thai) and Papua New Guinea (PNG).

the number of repeat units, two out of eleven isolates (Ven-IS9 and PNG Muz37) have 7 versus 6 repeats (Figure 2 ). No other polymorphisms were detected outside the repeat region

\section{In vitro inhibition of sporozoite invasion assay}

The results showed that the IgG against the non-repeatcontaining MB2-B peptide has the most potent inhibitory effect, 57\% (Table 4). In contrast, the IgG against the repeat-containing peptide, $\mathrm{MB} 2-\mathrm{C}$, exhibited only $18 \%$ inhibition. Moreover the IgG fraction against another repeat-containing but non-antigenic peptide, MB2-FA, conferred 33\% inhibition.

\section{Discussion}

MB2 is a novel sporozoite surface protein, that is present at several stages of the parasite lifecycle albeit in different sub-cellular locations as described earlier [20]. It is composed of three distinct domains, an amino terminal basic domain, a central acidic domain and a carboxyl terminal GTP binding domain as shown in the schematic Figure 1A. The surface localization of MB2 at the sporozoite stage provides a potential target for an antibody mediated response. Within the MB2 basic domain three overlapping polypeptides, and one non-overlapping polypeptide spanning between the basic and acidic domains, and three more polypeptides covering the remainder of the acidic domain and a C-terminal polypeptide were initially generated as GST-His-tagged sandwich fusions and used to immunize rabbits. The immune rabbit IgG was used in an inhibition of sporozoite invasion (ISI) assay. Antibodies against MB2 B inhibited sporozoite invasion (57\%), whilst antibodies directed against MB-FA and MB2-C inhibited poorly (33\% and $18 \%$ respectively) as shown in

Table 4: Evaluation of in vitro inhibition of sporozoite invasion (ISI) by rabbit antibodies against different regions of MB2

\begin{tabular}{llcl}
\hline IgG & SPOROZOITES/WELL & MEAN \pm SDa & \% INHIBITIONb \\
\hline MB2-B & & & 0 \\
PRE-IMMUNE & $522,514,593$ & $543 \pm 35$ & $57 \%$ \\
MB2-B & $205,251,238$ & $231 \pm 19$ & 0 \\
MB2-Cc & $216,312,240$ & $256 \pm 40$ & $18 \%$ \\
PRE-IMMUNE & $252,177,198$ & $209 \pm 31$ & 0 \\
MB2-C & $472,485,438$ & $465 \pm 20$ & $33 \%$ \\
MB2-FAc & $263,354,306$ & $307 \pm 37$ & $97 \%$ \\
PRE-IMMUNE & $11,9,6$ & $8 \pm 2$ & 0 \\
MB2-FA & $280,350,325$ & $318 \pm 29$ & \\
mAb NFSI & & & \\
MEM-Control & & & \\
\hline
\end{tabular}

a SD, standard deviation

b Inhibition expressed as \% relative to pre-immune control culture (0\%), and it was calculated as follows: $100 \times[\mathrm{I}-(\mathrm{mean}$ number of invaded sporozoites in test culture/mean number of invaded sporozoites in control culture)]. Pre-immune antibodies used as negative control.

c These peptides contain amino acid repeat motifs.

dMEM Minimal essential medium. 
Table 4, suggesting that antibodies directed against this non-repeat region may be important in protection against infection. All five protected volunteers produced antiMB2 antibodies preferentially against the non-repeat $M B 2-B$ region, and the ISI assay showed that rabbit antiMB2-B antibodies are more effective than antibodies against other regions of MB2 at blocking sporozoite invasion of hepatocytes. It may be speculated that anti-MB2-B antibodies may contribute to the overall protective sterile immunity acquired by non-replicating metabolicallyactive sporozoite immunized volunteers. Immunoblot analyses of plasma from individuals in an area of highly seasonal transmission during a period of high transmission also demonstrated higher frequencies of anti-MB2-B antibodies among individuals who were not infected with $P$. falciparum as compared to those who were. However, these individuals also had higher levels of anti-MB2-C antibodies. In this preliminary limited study the sera were obtained at a single point in time and we cannot know whether either type of antibodies were associated with protection from infection in this population. It is very likely that the inoculum of infection in these individuals was much lower than that of the irradiated sporozoiteinfected individuals. The data suggest that individuals with intermittent natural $P$. falciparum exposure have $\mathrm{B}$ cell responses that include regions of the MB-2 antigen recognized by those protected individuals exposed to irradiated sporozoites. The qualitative and quantitative antibody responses to the area most strongly associated with protection in immunized volunteers (MB-2B) may be impaired in individuals in areas of seasonal transmission. There was no clear relationship between malaria symptoms and presence of antibodies to a specific region (MB$2 \mathrm{~B}$ or $\mathrm{MB}-2 \mathrm{C})$, but since presence of antibodies was assessed at the time of disease, we can conclude only that symptomatic and asymptomatic individuals in this area are able to mount antibody responses to $\mathrm{MB}-2 \mathrm{~B}$ and $\mathrm{MB}-$ $2 \mathrm{C}$. Further studies are required to characterize these responses in individuals who reside in areas of high-level, year-round $P$. falciparum transmission, and prospective studies required to assess the association of these antibodies with protection from infection and disease in endemic areas.

One of the striking observations is that protected volunteers were able to recognize preferentially the non-repeat $\mathrm{MB} 2-\mathrm{B}$ and to a lesser degree the repeat-including MB2-C and $-D$ regions that most naturally exposed persons in malaria-endemic areas recognize. The significance of such a qualitative difference in the antibody response is difficult to assess due to the limited sample size. It is known that at the optimal radiation dosage required to induce sterile immunity, the weakened sporozoite is not able to develop completely in the hepatocytes [30,31]. Since the stage-dependent cellular localization of MB2 is accompa- nied by differential proteolytic processing [20], it may be that MB2 is aberrantly processed and is misdirected onto the surface of infected cells. Alternatively, B-cell response may be due to the exposure 'dose' of sporozoites. Protected volunteers received hundreds to more than a thousand bites of infected and irradiated mosquitoes in order to acquire sterile immunity [13]. In contrast, in malaria endemic countries, exposed individuals receive on average less than 200 infective bites per year [32], and in the highland area of Kenya where the endemic serum samples were collected, the number of infective bites is likely to be much lower than this [33]. It is possible that, the quantitative difference of inoculated sporozoites is responsible for the difference observed in antibody recognition against MB2 between protected volunteers and persons living in endemic countries. However antibodies against MB2-B were more frequent in those without parasitaemia than those with parasitaemia in the malaria endemic area, consistent with the findings in the protected versus unprotected volunteers.

The antigenic regions in the basic domain of MB2 were analyzed for potential amino acid polymorphisms. The nucleotide sequence of the MB2 gene was determined for field isolates obtained from different malaria-endemic regions of the world. It was expected that samples derived from non-overlapping locales would provide the greatest opportunity to detect sequence variation in MB2. The analysis of the primary structure of amplified MB2 DNA fragments from different isolates of $P$. falciparum showed that antigenic variation is unlikely to be a factor contributing to the different antibody response against MB2. Except for variation in the number of repeat units, the antigenic region in the B domain of $\mathrm{MB} 2$ is absolutely conserved among laboratory strains and field isolates collected from different parts of the world as shown in Figure 2. The amino acid sequence conservation may reflect a functional constraint of the B domain since it is not only exposed on the surface of the sporozoite but also translocated into the nucleus of blood-stage parasites[20]. In our in vitro ISI study, antibodies to this conserved, antigenic region of MB2 can inhibit sporozoite invasion of hepatocytes. If these antibodies play a role in protective immunity in vivo, the finding that the antigenic region of $\mathrm{MB} 2$ is highly conserved suggests that it might make a good target for immune attack by antibodies since most, if not all, $P$. falciparum sporozoites would be recognized.

The Plasmodium parasite is genetically complex, and based on the malaria genome sequencing projects [34] may have $5,000-6,000$ genes. Its antigenic composition also is expected to be complex. Thus, the challenge in designing effective malaria vaccine(s) is to define the immunogenic molecules that are essential and the methods to present them properly to the immune system to induce the 
desired immune responses that protected the volunteers experimentally immunized with the non-replicating metabolically-active sporozoite vaccine. The MB2 protein possesses a number of molecular and immunogenic properties that indicate it is an intriguing candidate to complement current vaccine studies. Studies have shown that immunity to malaria also is mediated, at least partly, by cellular immune mechanisms [35]. In endemic areas, cytotoxic T lymphocytes (CTLs) from exposed individuals recognize epitopes in a number of pre-erythrocytic antigens of $P$. falciparum, and indirect evidence indicates that these CTLs may play a role in protective immunity [3638]. Since MB2 was shown to be present in multiple developmental stages including the hepatic stage that can be recognized by CTLs, it will be important to obtain evidence that MB2 also is recognized by CTLs from P. falciparum-exposed individuals. The variation in the nature and strength of immune response observed in the endemic plasma samples may also be due to host factors such as MHC class I and II restriction. Further studies across multiple populations are needed to assess the type and strength of responses in individuals of differing genetic backgrounds. It is anticipated that as more novel parasite immunogens are characterized, the knowledge gained from studying them will help bridge the gap between recombinant and attenuated sporozoite vaccines.

The P. falciparum antigen MB2 is a multi-domain sporozoite surface protein [20]. In this study denatured recombinant peptide fragments MB2 were probed with immune plasma or sera resulting in the detection of antibodies directed against linear epitopes. Immunoblot analyses using serum of a volunteer protected by the exposure to non-replicating metabolically active sporozoites (\#5) revealed that linear epitopes within the basic domain of MB2 are recognized strongly, whilst the acidic domain is recognized poorly. In comparison, plasma from a person living in an endemic region (KU 162) contained antibodies against linear epitopes within both basic and acidic domains. Antibodies against linear epitopes within the GTP binding domain were not detected in either set of samples. Moreover, sera from all the protected volunteers (non-replicating, metabolically-active sporozoite immunized) contain antibodies against MB2. In contrast, no anti-MB2 antibodies were detected in sera of non-protected (also non-replicating metabolically-active sporozoite immunized) volunteers. Furthermore, antibodies from the serum of protected volunteers recognized preferentially the non-repeat-containing MB2-B peptide, while the repeat-containing MB2-C peptide is recognized preferentially by the antibodies in the plasma of persons living in malaria-endemic areas. In addition, although the acidic domain also contains two amino acid repeat regions [20], serum from a protected volunteer showed minimal antibody reactivity against the acidic domain. In contrast, two regions of the acidic domain, one of which contains amino acid repeats, were recognized strongly by the antibodies in the endemic plasma (MB2-D \& MB2-E). These qualitative results are interpreted to indicate that there may be one or more B-cell epitopes encoded in the nonrepeat regions of MB2-B, that are more relevant to protective immunity than those encoded in the repeat regions. The results of the inhibition of sporozoite invasion assay suggest that antibodies directed against epitopes encoded in the non-repeat region (MB2-B) possess greater anti-parasitic activities than antibodies against epitopes encoded in the repeat-included region (MB2-C). The in vitro ISI assay showed that anti-MB2-B antibodies are more effective than anti-MB2-FA and anti-MB2-C antibodies at blocking sporozoites from invading the hepatocyte. Although $\sim 40 \%$ of the parasites are still able to enter HepG2 cells, it is not known whether their intra-hepatic development is affected. The change of the cellular location of MB2 from the surface to the nucleus as the parasite lifecycle progresses from the sporozoite stage to the erythrocytic stage is consistent with the interpretation that it may have a function in the development of the parasite [20]. Thus, it is possible that anti-MB2 antibodies, although they partially block invasion of the sporozoites, they could also hinder the intra-hepatic development.

\section{Conclusion}

The multi-domain MB2 protein is a sporozoite surface antigen identified on the human malaria parasite,Plasmodium falciparum. Analysis of serum from eight human volunteers that were immunized via the bites of $P$. falciparum infected irradiated mosquitoes, revealed five that developed immunity and were completely protected against subsequent challenge with non-irradiated parasite also had detectable levels of antibody against MB2. In contrast, in three volunteers not protected, anti-MB2 antibodies were below the level of detection. Moreover, anti-MB2 antibodies were detected in the plasma of $83 \%$ of the individuals living in a malaria endemic area of Kenya $(n=41)$. Antibodies from protected volunteers preferentially recognized a non-repeat region of the basic domain of MB2, whereas plasma from naturally-infected individuals had antibodies that recognize regions of MB2 that contain a repeat motif. Furthermore, rabbit polyclonal antibodies targeting the non-repeat region of the basic domain conferred greater inhibition of sporozoites entry into HepG2A16 cells in vitro relative to antibodies directed against the repeat regions. Sequence analysis of eleven field isolates and four laboratory strains showed that these antigenic regions of the $\mathrm{B}$ domain of the $\mathrm{MB} 2$ gene are highly conserved in parasites obtained from different parts of the world. A preliminary analysis of the human humoral response against MB2 indicates that it may be an additional conserved target for immune intervention at the pre-erythrocytic stage of $P$. falciparum life cycle. 


\section{Competing interests}

The authors declare that they have no competing interests.

\section{Authors' contributions}

TVN constructed the recombinant plasmids, expressed and purified the proteins and carried out the affinity purification of the rabbit antibodies, immunoblot analysis and DNA sequencing analysis. JBS and PdIV carried out the inhibition of sporozoite invasion assay. CCJ co-ordinated the collection of serum and plasma via KMRI.

AAJ and ASK initiated the study and supervised TVN. All authors contributed to data analysis and drafting of the manuscript.

\section{Acknowledgements}

This work was supported by grants from the Burroughs-Wellcome Fund and the John D. and Catherine T. MacArthur Foundation (to AAJ), NIH Al0I572 (to CCJ), and by the Naval Medical Research and Development Command work units STO F 6.I 6II02AAOI00BFX and STO F 6.2 62787A00IOIEFX (to JBS).

The authors are grateful to W.O. Rogers (US Naval Medical Research Center, Rockville), U. Krzych and I. Chalom (Walter Reed Army Institute of Research, Washington DC) for supplying serum samples from irradiatedsporozoite immunized volunteers, and A. Lal (Centers for Disease Control and Prevention, Atlanta) for providing parasite genomic DNA samples. The experiments reported herein were conducted according to the principles set forth in the "Guide for the Care and Use of Laboratory Animals," Institute of Laboratory Animals Resources, National Research Council,

National Academy Press, 1996. The assertions herein are the private ones of the authors and are not to be construed as official or as reflecting the views of the United States Navy or the Naval service at large. This paper was published with the permission of the Director of the Kenya Medical Research Institute.

\section{References}

I. Aregawi M, Cibulskis R, Otten M, Williams R, Dye C: World Malaria Report 2008. In WHO Global Malaria Programme Edited by: Aregawi M, Cibulskis R, Otten M, Williams R, Dye C. Geneva: World Health Organisation; 2008.

2. Ballou WR, Arevalo-Herrera M, Carucci D, Richie TL, Corradin G, Diggs C, Druilhe P, Giersing BK, Saul A, Heppner DG, et al: Update on the clinical development of candidate malaria vaccines. Am J Trop Med Hyg 2004, 7 I (2 Suppl):239-247.

3. Greenwood B: Malaria vaccines. Evaluation and implementation. Acta Trop 2005, 95:298-304.

4. Greenwood BM, Bojang K, Whitty CJ, Targett GA: Malaria. Lancet 2005, 365(9469): I 487-1498.

5. Moorthy VS, Good MF, Hill AV: Malaria vaccine developments. Lancet 2004, 363:150-156.

6. Hill AV: Pre-erythrocytic malaria vaccines: towards greater efficacy. Nat Rev Immunol 2006, 6:2I-32.

7. Clyde DF, Most H, McCarthy VC, Vanderberg JP: Immunization of man against sporozite-induced falciparum malaria. Am J Med Sci 1973, 266:169-177.

8. Bojang KA, Milligan PJ, Pinder M, Vigneron L, Alloueche A, Kester KE, Ballou WR, Conway DJ, Reece WH, Gothard P, et al.: Efficacy of RTS, S/ASO2 malaria vaccine against Plasmodium falciparum infection in semi-immune adult men in The Gambia: a randomised trial. Lancet 200I, 358:1927-1934.

9. Luke TC, Hoffman SL: Rationale and plans for developing a nonreplicating, metabolically active, radiation-attenuated Plasmodium falciparum sporozoite vaccine. J Exp Biol 2003, 206(Pt 2I):3803-3808.
10. Dame JB, Williams JL, McCutchan TF, Weber JL, Wirtz RA, Hockmeyer WT, Maloy WL, Haynes JD, Schneider I, Roberts D, et al.: Structure of the gene encoding the immunodominant surface antigen on the sporozoite of the human malaria parasite Plasmodium falciparum. Science 1984, 225(4662):593-599.

II. Yoshida N, Nussenzweig RS, Potocnjak P, Nussenzweig V, Aikawa M: Hybridoma produces protective antibodies directed against the sporozoite stage of malaria parasite. Science 1980, 207:7|-73.

12. Zavala F, Cochrane AH, Nardin EH, Nussenzweig RS, Nussenzweig V: Circumsporozoite proteins of malaria parasites contain a single immunodominant region with two or more identical epitopes. J Exp Med 1983, 157:1947-1957.

13. Herrington D, Davis J, Nardin E, Beier M, Cortese J, Eddy H, Losonsky G, Hollingdale M, Sztein M, Levine M, et al:: Successful immunization of humans with irradiated malaria sporozoites: humoral and cellular responses of the protected individuals. Am J Trop Med Hyg 1991, 45:539-547.

14. Chappel JA, Hollingdale MR, Kang AS: IgG(4) Pf NPNA-I a human anti-Plasmodium falciparum sporozoite monoclonal antibody cloned from a protected individual inhibits parasite invasion of hepatocytes. Hum Antibodies 2004, 13:91-96.

15. Chappel JA, Rogers WO, Hoffman SL, Kang AS: Molecular dissection of the human antibody response to the structural repeat epitope of Plasmodium falciparum sporozoite from a protected donor. Malar J 2004, 3:28.

16. Pinder M, Reece $\mathrm{WH}$, Plebanski M, Akinwunmi P, Flanagan KL, Lee EA, Doherty T, Milligan P, Jaye A, Tornieporth N, et al.: Cellular immunity induced by the recombinant Plasmodium falciparum malaria vaccine, RTS, S/AS02, in semi-immune adults in The Gambia. Clin Exp Immunol 2004, 135:286-293.

17. Robson KJ, Hall JR, Jennings MW, Harris TJ, Marsh K, Newbold Cl, Tate VE, Weatherall DJ: A highly conserved amino-acid sequence in thrombospondin, properdin and in proteins from sporozoites and blood stages of a human malaria parasite. Nature 1988, 335:79-82.

18. Rogers WO, Malik A, Mellouk S, Nakamura K, Rogers MD, Szarfman A, Gordon DM, Nussler AK, Aikawa M, Hoffman SL: Characterization of Plasmodium falciparum sporozoite surface protein 2. Proc Natl Acad Sci USA 1992, 89:9176-9180.

19. Fidock DA, Bottius E, Brahimi K, Moelans II, Aikawa M, Konings RN, Certa U, Olafsson P, Kaidoh T, Asavanich A, et al.: Cloning and characterization of a novel Plasmodium falciparum sporozoite surface antigen, STARP. Mol Biochem Parasitol 1994, 64:219-232.

20. Nguyen TV, Fujioka $H$, Kang AS, Rogers WO, Fidock DA, James AA: Stage-dependent localization of a novel gene product of the malaria parasite, Plasmodium falciparum. J Biol Chem 200I, 276:26724-2673I.

21. Romero LC, Nguyen TV, Deville B, Ogunjumo O, James AA: The MB2 gene family of Plasmodium species has a unique combination of SI and GTP-binding domains. BMC Bioinformatics 2004, 5:83.

22. Verra $F$, Hughes AL: Biased amino acid composition in repeat regions of Plasmodium antigens. Mol Biol Evol 1999, 16:627-633.

23. Wrightsman RA, Dawson BD, Fouts DL, Manning JE: Identification of immunodominant epitopes in Trypanosoma cruzi trypomastigote surface antigen-I protein that mask protective epitopes. J Immunol I994, I 53:3 I 48-3 I54.

24. Stratmann T, Schmid SR, Harper JF, Kang AS: Bacterial expression and purification of recombinant Plasmodium yoelii circumsporozoite protein. Protein Expr Purif 1997, I I:72-78.

25. John CC, Zickafoose JS, Sumba PO, King CL, Kazura JW: Antibodies to the Plasmodium falciparum antigens circumsporozoite protein, thrombospondin-related adhesive protein, and liver-stage antigen $I$ vary by ages of subjects and by season in a highland area of Kenya. Infect Immun 2003, 7 I:4320-4325.

26. John CC, Sumba PO, Ouma JH, Nahlen BL, King CL, Kazura JW: Cytokine responses to Plasmodium falciparum liver-stage antigen I vary in rainy and dry seasons in highland Kenya. Infect Immun 2000, 68:5 I 98-5204.

27. Mellouk S, Berbiguier N, Druilhe P, Sedegah M, Galey B, Yuan L, Leef $M$, Charoenvit $Y$, Paul C, Hoffman S, et al.: Evaluation of an in vitro assay aimed at measuring protective antibodies against sporozoites. Bull World Health Organ 1990, 68(Suppl):52-59. 
28. Charoenvit $Y$, Fallarme $V$, Rogers WO, Sacci JB Jr, Kaur M, Aguiar JC, Yuan LF, Corradin G, Andersen E, Wizel B, et al.: Development of two monoclonal antibodies against Plasmodium falciparum sporozoite surface protein 2 and mapping of B-cell epitopes. Infect Immun 1997, 65:3430-3437.

29. Hollingdale MR, Nardin EH, Tharavanij S, Schwartz AL, Nussenzweig RS: Inhibition of entry of Plasmodium falciparum and $P$. vivax sporozoites into cultured cells; an in vitro assay of protective antibodies. J Immunol 1984, I 32:909-9|3.

30. Sigler $\mathrm{Cl}$, Leland $\mathrm{P}$, Hollingdale $\mathrm{MR}$ : In vitro infectivity of irradiated Plasmodium berghei sporozoites to cultured hepatoma cells. Am J Trop Med Hyg 1984, 33:544-547.

31. Silvie O, Semblat JP, Franetich JF, Hannoun L, Eling W, Mazier D: Effects of irradiation on Plasmodium falciparum sporozoite hepatic development: implications for the design of preerythrocytic malaria vaccines. Parasite Immunol 2002, 24:22I-223.

32. Hay SI, Rogers DJ, Toomer JF, Snow RW: Annual Plasmodium falciparum entomological inoculation rates (EIR) across Africa: literature survey, Internet access and review. Trans $R$ Soc Trop Med Hyg 2000, 94: I I3-I27.

33. Khaemba BM, Mutani A, Bett MK: Studies of anopheline mosquitoes transmitting malaria in a newly developed highland urban area: a case study of Moi University and its environs. East Afr Med J 1994, 71:159-164.

34. Gardner MJ, Tettelin H, Carucci DJ, Cummings LM, Smith HO, Fraser CM, Venter JC, Hoffman SL: The malaria genome sequencing project: complete sequence of Plasmodium falciparum chromosome 2. Parassitologia 1999, 41:69-75.

35. Hoffman SL, Franke ED, Hollingdale MR, Druilhe P: Attacking the infected hepatocyte. In Malaria vaccine Development Edited by: Hoffman SL. Washington, DC: American Society for Microbiology; 1996:35.

36. Aidoo M, Lalvani A, Allsopp CE, Plebanski M, Meisner SJ, Krausa $P$, Browning M, Morris-Jones S, Gotch F, Fidock DA, et al.: Identification of conserved antigenic components for a cytotoxic $T$ lymphocyte-inducing vaccine against malaria. Lancet 1995, 345: $1003-1007$.

37. Aidoo M, Lalvani A, Gilbert SC, Hu JT, Daubersies P, Hurt N, Whittle HC, Druihle P, Hill AV: Cytotoxic T-lymphocyte epitopes for HLA-B53 and other HLA types in the malaria vaccine candidate liver-stage antigen 3. Infect Immun 2000, 68:227-232.

38. Aidoo M, Lalvani A, Whittle HC, Hill AV, Robson KJ: Recombinant vaccinia viruses for the characterization of Plasmodium falciparum -specific cytotoxic $\mathbf{T}$ lymphocytes: recognition of processed antigen despite limited re-stimulation efficacy. Int Immunol 1997, 9:731-737.

39. Sambrook JF, Russell DW: Molecular Cloning: A Laboratory Manual. 3rd edition. Cold Spring Harbor Laboartory Press; 2001.

Publish with Bio Med Central and every scientist can read your work free of charge

"BioMed Central will be the most significant development for disseminating the results of biomedical research in our lifetime. "

Sir Paul Nurse, Cancer Research UK

Your research papers will be:

- available free of charge to the entire biomedical community

- peer reviewed and published immediately upon acceptance

- cited in PubMed and archived on PubMed Central

- yours - you keep the copyright

Submit your manuscript here:

http://www.biomedcentral.com/info/publishing_adv.asp
BiolMedcentral 